\title{
Study on Operation Control Strategy of Single Stage Pumping Station in Long - distance and Multi - stage Pumping Station Control System
}

\author{
Xu Xuejun ${ }^{1, a}$, Ma qingqing ${ }^{2, b}$, Yan Ying ${ }^{3, c}$ \\ ${ }^{1}$ School of Hydropower and Information Engineering,Huazhong University of Science and \\ Technology, Wuhan, Hubei 430074, P. R. China \\ ${ }^{2}$ School of Hydropower and Information Engineering,Huazhong University of Science and \\ Technology, Wuhan,Hubei 430074, P. R. China \\ ${ }^{3}$ Patent Examination Cooperation Hubei Center of the Patent Office, SIPO, \\ Wuhan,Hubei 430205, China \\ ${ }^{a}$ xxj_hust@hust.edu.cn
}

Key words: Long-distance water delivery. single-stage pumping station. power-on scheme.

Abstract. Aiming at the optimal operation of single-stage pumping station in the multi-stage pumping station with small buffer pool, on the basis of the research work at the present stage of long-distance multi-stage pumping stations, the objective function, Head flow and other constraints within the single-stage pumping station optimization model, using the penalty function of the genetic algorithm to calculate the level of the pump station under the designated flow, including the boot state, the frequency control pump boot speed control strategy, To provide a theoretical basis and data support for the joint control of long buffer multistage pumping stations in small buffer pools.

\section{Research background}

The operation control of long-distance multistage pump station project in our country is divided into two levels, namely adjacent-stage pumping station and single-stage pumping station. At present, domestic and foreign experts and scholars have made a series of achievements in the optimization control of single-stage pumping station, Nitivattananon [1] proposed a dynamic programming-based optimization model to meet the requirements of real-time scheduling operation of single-stage pump stations, which can effectively reduce the number of on and off times and save $20 \%$ of annual operating costs. SrinivaSa Lingireddy [2] Aiming at the single-stage pumping station operation, taking the highest operating efficiency as the goal, the genetic algorithm was used to optimize the pump speed and quantitatively calculate the water-lifting cost saved by the variable speed regulation. Zheng $\mathrm{Wu}$ [3] The single-stage pumping station has been optimized and the optimization of the flow, lift and opening and shut-down during the period has been optimized to obtain the maximum operating efficiency. The problems in the single-stage pumping station are mainly that some pumping stations are inefficient, Due to the dynamic changes of operating conditions in the process of water delivery, the pumping station scheduling can not be based on real-time flow, lift changes at all levels of pumping station within the pump unit shipped Real-time adjustment of the program line, resulting in deviation from the high-efficiency zone, resulting in low operating efficiency pumping station, water transport costs. This article aims at optimizing the operation of single-stage pumping station, and formulating the corresponding joint scheduling and control scheme to achieve the goal of efficient and economical operation of the system.

\section{Internal optimization of pumping station mathematical modeling}

The problem of single-stage pumping station optimal operation is mainly to determine the number of (Note: This paper has been supported by the Innovative Rearch Fund of HUST)

units and the frequency of the variable frequency pump under the given distribution flow. The principle of starting the unit is to put the unit into operation, while meeting the pump flow requirements while minimizing the operating costs. For pumping stations with multiple units, the 
total pumping capacity is the sum of the pumping capacity of each operating unit. Currently for long-distance large-scale water diversion pumping station traffic changes in demand, the main control method is "the number of start-up station + variable speed pump speed regulation." Among them, the number of start-up machines plays the role of rough adjustment in stages, and the variable speed pump's speed regulation achieves a relatively continuous adjustment in the section. During operation, the combination of the constant speed pump and the speed regulating pump is determined according to the required flow rate.

pump curve fitting.Accurate description of pump performance curve is a prerequisite for establishing mathematical model of pumping station optimal scheduling problem. The pump head expression, shaft power expression can be obtained from the product curve of each pump fitting. Pump at rated speed flow and lift the following:

$$
H_{i}=H_{i 0}-S_{i} Q_{i}^{2}
$$

Set the pump shaft power at rated speed as quadratic polynomial flow, namely:

$$
P_{i}=b_{i 0}+b_{i 1} Q_{i}+b_{i 2} Q_{i}^{2}=\Sigma_{j=0}^{2}\left(b_{i j} Q_{i}\right)
$$

According to the principle of fluid mechanics, the expression of pump head and shaft power of variable frequency pump at non-rated speed is respectively:

$$
\begin{aligned}
& H_{i}=k_{i}{ }^{2} H_{i 0}-S_{i} Q_{i}{ }^{2} \\
& P_{i}=b_{i 0} k_{i}{ }^{3}+b_{i 1} k_{i}{ }^{2} Q_{i}+b_{i 2} k_{i} Q_{i}{ }^{2}=\sum_{j=0}^{2}\left(b_{i j} k_{i}{ }^{3-j} Q_{i}{ }^{j}\right) \\
& k_{i}=\frac{n_{i}}{n_{i Q}}
\end{aligned}
$$

$n_{i}(\mathrm{r} / \mathrm{min})$ : the unit working speed of pump i; $n_{i \mathrm{o}}$ : the unit rated speed of pump I; $k_{i}$ : speed adjustment; $P_{i}(\mathrm{KW})$ : Pump shaft power; $Q_{i}:\left(\mathrm{m}^{3} / \mathrm{s}\right)$ Pump flow; $b_{i 0}, b_{i 1}, b_{i 2}$ : Pending factor in pump shaft power expression; $H_{i}$ : Pump head; $S_{i}$ :Deficiency coefficient of resistance; $H_{i 0}$ :Virtual head.

Optimization Model.The optimization mathematical model can be generally described as an objective function and a constraint function, and its general form is:

(1) objective function

The optimal operation target of this pump station unit is running power consumption minimum [4], so assuming that the pump station is equipped with a total of $n$ pumps, including $m$ variable frequency pump, the rest are constant speed pump, then the time The objective function of pump station is:

$$
\begin{aligned}
& \min U=\min \left[\sum_{i=1}^{n} x_{i} P_{1}\right] \\
& \sum_{i=1}^{n} x_{i} P_{i}=\sum_{i=1}^{m} x_{i}\left[\sum_{j=0}^{2}\left(b_{i j} k_{i}{ }^{3-j} Q_{i}{ }^{j}\right)\right]+\sum_{i=m+1}^{n} x_{i}\left[\sum_{j=0}^{2}\left(b_{i j} Q_{i}^{j}\right)\right]
\end{aligned}
$$

\section{(2) Constraints}

Pump unit stand-alone flow constraints

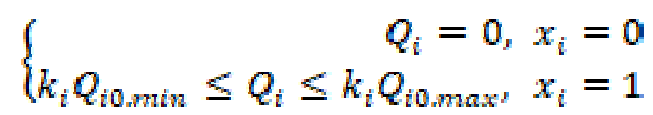

Total traffic constraints:

$$
Q=\sum_{i=1}^{n} x_{i} Q_{i}
$$


Head restraint: V

$$
H=k_{i}^{2} H_{i 0}-S_{i} Q_{i}^{2}
$$

Frequency conversion pump frequency constraints and Put into operation the number of constraints:

$$
\begin{aligned}
& 0.5 n_{i 0} \leq n_{i} \leq n_{i 0} \\
& 0<\sum_{i=1}^{n} x_{i} \leq n
\end{aligned}
$$

$\mathrm{U}(\mathrm{KW})$ : The total power consumption of all pumps in operation at a given flow rate ; $x_{i}$ : State function; $x_{i}=0$ : Pump down; $x_{i}=1$ : Pump operation; Q iamin, $Q_{\text {iamax }}$ : Pump at the rated speed of the most efficient area of the next flow and maximum flow; Q: The pumping station of this level of water flow requirements; For fixed speed pumps, $k_{i}=1$;

\section{Single-stage pumping station optimization model solution}

In the mathematical model established in the second part of this paper, both the continuous variable pump speed $k_{i}$ and the discrete variable pump startup state $x_{i}$ are included, and the existing and the inequality constraints in the constraints are both linear programming, Dynamic programming [5] [6] [7] are more complicated to solve. For multi-peak function and morbid function, the optimal solution obtained by the general optimization algorithm is often only the local optimal solution, it is difficult to get the global optimal solution. Genetic algorithm is an optimization algorithm that has been proved to be able to converge to the global optimal solution of the problem with a high probability. Therefore, this paper will also use this method to solve the single-stage pumping station optimization operation under a given flow rate.

In actual application, the common single-stage pump station optimizes the economic cost and the control cost of the variable-speed pump during operation, so most single-stage pump stations are equipped with one variable-frequency pump under normal circumstances. So suppose a single-stage pumping station is equipped with four pumps, of which only one variable-frequency pump, the remaining three are constant-speed pump, so this article in the mathematical model described in the previous chapter is based on the assumption that $\mathrm{m}=1, \mathrm{~N}=4$ under the premise of the solution. Then the objective function $\mathrm{U}$ at this time can be simplified as the following expression:

$$
U=\sum_{i=1}^{n} x_{i} P_{i}=x_{1} \Sigma_{j=0}^{2}\left(b_{1 j} k^{3-j} Q_{1}{ }^{j}\right)+\sum_{i=2}^{4}\left[x_{i} \Sigma_{j=0}^{2} b_{i j} Q_{i}^{j}\right]
$$

At this point the main constraints are as follows:

Total pumping station constraints: $Q=\sum_{i=1}^{n} x_{i} Q_{i}$

Pumping station head restraint: $H=k_{i}^{2}{ }^{2} H_{i 0}-S_{i} Q_{i}{ }^{2}$

Frequency conversion pump frequency constraints: $0.5 \leq k \leq 1$

\section{Genetic Algorithm to solve}

(1) coding method

The above mathematical model shows that the design variables are the state function $X_{i}$ and the rate $\mathrm{k}$. The unit state function $X_{i}$ is a discrete variable, binary coding can be used directly, while the rate $\mathrm{k}$ for the continuous variable, you need to go through the discretization and then use binary coding. In this paper, a 6-bit binary number to adjust the rate $\mathrm{k}$ encoding, combined with the transfer rate constraints $\left(0.5 n_{i 0} \leq n_{i} \leq n_{i 0}\right)$, The rate of change $\mathrm{k}$ in the range of 0.5 to 1 is divided into 64 intervals to achieve the purpose of higher accuracy, After determining the coding rules, we can confirm the structure of the chromosome, $\frac{x_{1} x_{2} x_{2} x_{4}}{4}+\frac{k_{1} k_{3} k_{3} k_{4} k_{3} k_{5}}{3}$, Chromosome length of a total of ten. After determining the coding mode, the initial population should be considered. The initial population is 
generated by the random number generator. In order to ensure the optimal solution, the initial population size is taken as 100 .

(2) penalty function

The optimization problem solved in this paper is that both the equality constraint and the inequality constraint, for the total pumping station $Q=\sum_{i=1} x_{i} Q_{i}$ equality constraint, this paper uses the penalty function method to solve, Set penalty function to:

$$
F=\left(Q-\sum_{i=1}^{4} Q_{i}\right)^{2}
$$

According to the formula (2-3) in the head and the relationship between the flow rate can be drawn for each pumping station unit flow is:

$$
Q_{i}=\sqrt{\frac{H-k^{2} H_{i n}}{s_{i}}}
$$

According to the above fitness function, the fitness value w (f) of each individual in the maternal population can be calculated. Then, the fitness of each individual can be calculated according to the eligibility calculation formula of this generation:

$$
u_{i}=1-\frac{w_{i}(f)}{w_{\max }(f)+w_{\min }(f)}
$$

M: Penalty factor; U: Pump station unit shaft power and the sum

(3) choice, cross, mutation operation

The algorithm uses a single-point crossover random cross-point method, the intersection rate of 0.4; mutation although can produce more new individuals, but also may undermine the original good higher individual, so its value should not be over Large, this variation rate taken 0.08.

(4) Determination of convergence conditions

In this paper, we use the method of terminating algebra and convergence inequality to judge whether the optimal solution is reached. The ending algebra $\mathrm{T}$ is set to 200 , the discriminant inequality is:

$$
\frac{|u(G+1)-u(c)|}{u(G)} \leq 0.01
$$

$\mathrm{u}(\mathrm{G})$ : The best individual in the population is fine

Genetic Algorithm to solve the example.According to the example of a water conveyance project, it is assumed that a certain pumping station has a flow requirement of $0.45 \mathrm{~m}^{2} / \mathrm{s}$ during a certain period of time and a pumping station device has a lift requirement of $40 \mathrm{~m}$. The parameter list of the pumping station unit is shown in the following table:

\begin{tabular}{lccccc}
\hline model & $\begin{array}{c}\text { Limited } \\
\left(\boldsymbol{m}^{\mathbf{3}} / \mathbf{s}\right)\end{array}$ & $\begin{array}{c}\text { Data } \\
(\mathbf{m})\end{array}$ & $\begin{array}{c}\text { Rated } \\
(\mathbf{k W})\end{array}$ & $\begin{array}{c}\text { head } \\
\text { ( }\end{array}$ & $\begin{array}{c}\text { pated } \\
(\mathbf{r} / \mathbf{m i n})\end{array}$ \\
\hline $\mathbf{3 0 0 S 5 8}$ & 0.2195 & 58 & 200 & 1450 \\
$\mathbf{2 0 0 S 4 2}$ & 0.0778 & 42 & 37.8 & 2950 \\
$\mathbf{2 5 0 S 6 5}$ & 0.1300 & 54 & 132 & 1450 \\
\hline
\end{tabular}

The fixed speed pump in operation its head and power fitting expressions are:

$$
\begin{aligned}
& H=57.3-2504.09 Q^{2} \\
& P=9.512+771.4 Q-7560 k Q^{2}
\end{aligned}
$$


Use the above genetic algorithm to solve the boot program and operating conditions at this time as shown in the following table:

\begin{tabular}{lllll}
\hline Unit number & Open state & $\begin{array}{l}\text { Speed } \\
\text { adjustment }\end{array}$ & flow $\left(\boldsymbol{m}^{3} / \mathbf{s}\right)$ & $\begin{array}{c}\text { Shaft power } \\
(\mathbf{k W})\end{array}$ \\
\hline $\begin{array}{l}\text { Variable } \\
\text { speed pump }\end{array}$ & $\mathrm{Y}$ & 0.758 & 0.1664 & 87.11 \\
$\mathbf{1}$ & & & \\
$\begin{array}{l}\text { Constant } \\
\text { speed pump }\end{array}$ & $\mathrm{Y}$ & 1 & 0.0778 & 37.80 \\
$\mathbf{2}$ & & & \\
$\begin{array}{l}\text { Constant } \\
\text { speed pump }\end{array}$ & $\mathrm{Y}$ & & 0.0778 & 37.80 \\
$\mathbf{3}$ & 1 & & \\
$\mathbf{C}$ & & & 132.0 \\
Constant & $\mathrm{Y}$ & 1 & 0.1300 & \\
$\mathbf{4}$ & & & \\
\hline
\end{tabular}

\section{Conclusions}

Based on the research work at the present stage of long distance multi-stage pump station water conveyance project, the problems of single-stage pumping station optimal operation are studied and analyzed. Based on the principle of pump speed control, the lift-flow and shaft power-flow curve fitting of variable speed pump, Then, a system model with single-stage pumping station under the specified flow rate and the power of the system shaft as the objective function and with the speed at the flow-on state as the constraint condition is established by using the optimization theory. The model solver uses the genetic algorithm commonly used in pump station optimal scheduling. First, the inequality constraint of speed ratio is transformed into the coding strategy of variable, and then the equation constraint of flow constraint is transformed into the penalty function. After solving the constraints, the power-on scheme in a single-stage pumping station is obtained according to the basic genetic algorithm solving process.

\section{References}

[1] VilaS Nitiva_ttananon, Elaine C. Sadowski, Rafael G. . Qunimpo. Optimization of Water Supplyoperation. Water Resources Planning and MaIlagement, 1996, 122(5): 374 384.

[2] SrinivaSa Lingireddy, Don J. Wood. Improved Operation of Water Distribution Systems using Vauriable-Speed Pumps[J].Joumal of Energy Engineering,1998， 24(3): 90 103.

[3] Zheng Wu, Paul Boulos, Wemer de Schaetzen, etc.Using Genetic Algorithms for Water Distribution System Optimization.World Water Congress,2001.

[4] Thomas Walski. Development of water resources planning and management[J]. of Water Resources Planning and Management, 2001,126(4):203-205.

[5] Vieira F, Ramos H M. Optimization of operational planning for wind/hydro hybrid water supply systems [J].Renewable Energy, 2009, 34(3): 928-936. 
[6] Theocharisa M E, Tzimopoulosb C D, Sakellariou-Makrantonakic M A, et al. Comparative calculation of irrigation networks using Labye's method, the linear programming method and a simplified nonlinear method[J]. Mathematical and Computer Modeling, 2010,51(3/4): 286299.

[7] Staden AJV, Zhang J F, Xia X H. A model predictive control strategy for load shifting in a water pumping scheme with maximum demand charges [J]. Applied Energy, 2011, 88(12): 47854794. 